\title{
Heavy Quark Spectroscopy
}

\author{
Daniele Fasanella ${ }^{a}$, Andrea Ferretto Parodi $^{b}$ and Marco Pappagallo ${ }^{c}$ \\ ${ }^{a}$ University of Bologna and INFN \\ ${ }^{b}$ University of Genova and INFN \\ ${ }^{c}$ University of Glasgow
}

\begin{abstract}
Spectroscopy of hadrons containing heavy quarks is an important part of the LHC physics program. Precise measurements of observables like hadron masses are crucial to test the validity of QCD models, and at the same time they give the opportunity to search for new particles and previously unobserved decay modes. A large amount of results in hadronic spectroscopy has been already produced by ATLAS, CMS and LHCb experiments with data collected at $\sqrt{s}=7$ $\mathrm{TeV}$. This review summarizes the most relevant measurements of heavy hadrons masses, many of which are already more precise than the previous world best values. Particular emphasis is given to the first observations of new particles, and to the interpretation of some controversial exotic states.
\end{abstract}

VI Italian workshop on p-p physics at the LHC,

8-10 May 2013

Acquario di Genova, Ponte Spinola, Area Porto Antico, Genova, Italy 


\section{Introduction}

Heavy hadron spectroscopy is of great interest in the LHC physics program. The different QCD theories strongly depend on observables related to hadrons containing heavy quarks (like masses, lifetimes, quantum numbers, ...), and precise measurements of such quantities give the opportunity to test the models and constrain their parameters. Furthermore, the concrete possibility to observe new particles or decay channels would improve the current theoretical knowledge.

This letter presents a summary of the current measurements made by the ATLAS, CMS and LHCb experiments. Most of the these measurements are made with the data collected in 2010 and 2011 at $\sqrt{s}=7 \mathrm{TeV}$.

\section{Mass measurements}

In the open charm sector, $\mathrm{LHCb}$ has measured the $D$ meson mass differences using $1 \mathrm{fb}^{-1}$ of data collected at $\sqrt{s}=7 \mathrm{TeV}$ [1]. The measured mass differences are $m\left(D^{+}\right)-m\left(D^{0}\right)=$ $4.76 \pm 0.12$ (stat) \pm 0.07 (syst) $\mathrm{MeV}$ and $m\left(D_{s}^{+}\right)-m\left(D^{+}\right)=98.68 \pm 0.03$ (stat) \pm 0.04 (syst) $\mathrm{MeV}$. Precise determination of the $D^{0}$ mass is made using the decay mode $D^{0} \rightarrow K^{+} K^{-} K^{-} \pi^{+}$, measuring $m\left(D^{0}\right)=1864.75 \pm 0.15$ (stat) \pm 0.11 (syst) $\mathrm{MeV}$.

Measurements of the $\Lambda_{b}^{0}$ mass and lifetime have been published by ATLAS [2] and CMS [3]. Both analyses use about $5 \mathrm{fb}^{-1}$ of data collected at $\sqrt{s}=7 \mathrm{TeV}$ and the decay mode $\Lambda_{b}^{0} \rightarrow J / \psi(\rightarrow$ $\left.\mu^{+} \mu^{-}\right) \Lambda^{0}\left(\rightarrow p \pi^{-}\right)$. A simultaneous mass and decay time fit is used to extract the $\Lambda_{b}^{0}$ mass and lifetime. ATLAS measures $m\left(\Lambda_{b}^{0}\right)=5619.7 \pm 0.7$ (stat) \pm 1.1 (syst) $\mathrm{MeV}$ and $\tau\left(\Lambda_{b}^{0}\right)=1.449 \pm$ 0.036 (stat) \pm 0.017 (syst) ps, while CMS measures $m\left(\Lambda_{b}^{0}\right)=5619.7 \pm 0.5$ (stat) $\mathrm{MeV}$ and $\tau\left(\Lambda_{b}^{0}\right)=$ $1.503 \pm 0.052$ (stat) \pm 0.031 (syst) ps. The two sets of measurements are in good agreement.

Bottom baryons $\Lambda_{b}^{0}, \Xi_{b}^{-}$and $\Omega_{b}^{-}$masses have been measured by LHCb, with $1 \mathrm{fb}^{-1}$ of data collected at $\sqrt{s}=7 \mathrm{TeV}$ [4]. The reconstructed decay modes are $\Lambda_{b}^{0} \rightarrow J / \psi \Lambda^{0}\left(\rightarrow p \pi^{-}\right), \Xi_{b}^{-} \rightarrow$ $J / \psi \Xi^{-}\left(\rightarrow \Lambda^{0} \pi^{-}\right)$and $\Omega_{b}^{-} \rightarrow J / \psi \Omega^{-}\left(\rightarrow \Lambda^{0} K^{-}\right)$, where the $J / \psi$ is always reconstructed through $J / \psi \rightarrow \mu^{+} \mu^{-}$. The measured mass values are $m\left(\Lambda_{b}^{0}\right)=5619.44 \pm 0.13$ (stat) \pm 0.38 (syst) MeV, $m\left(\Xi_{b}^{-}\right)=5795.8 \pm 0.9$ (stat) \pm 0.4 (syst) $\mathrm{MeV}$ and $m\left(\Omega_{b}^{-}\right)=6046.0 \pm 2.2$ (stat) \pm 0.5 (syst) $\mathrm{MeV}$. All these measurements are the most precise to date.

Measurements related to the bottomonium $\Upsilon$ states have an important role in the flavour physics program of the LHC experiments. The states $\Upsilon(1 S), \Upsilon(2 S)$ and $\Upsilon(3 S)$ have been observed, in the $\mu^{+} \mu^{-}$final state, by all experiments with the early 2010 data. ATLAS, CMS and LHCb have measured the production cross section of the three states, using respectively $1.8 \mathrm{fb}^{-1}$ [5], $36 \mathrm{pb}^{-1}$ [6] and $25 \mathrm{pb}^{-1}$ [7] of data collected at $\sqrt{s}=7 \mathrm{TeV}$. CMS has measured also the polarizations of the three states with $4.9 \mathrm{fb}^{-1}$ of $\sqrt{s}=7 \mathrm{TeV}$ data [8], observing no evidence of large transverse or longitudinal polarizations in the explored kinematic region. The mass values measured by LHCb are $m(\Upsilon(1 S))=9448.3 \pm 0.5$ (stat) $\mathrm{MeV}, m(\Upsilon(2 S))=10010.4 \pm 1.4$ (stat) $\mathrm{MeV}$ and $m(\Upsilon(3 S))=10338.7 \pm 2.6($ stat $) \mathrm{MeV}$.

The $B_{c}^{+}$meson has been observed at the LHC through different final states. The decay mode $B_{c}^{+} \rightarrow J / \psi\left(\rightarrow \mu^{+} \mu^{-}\right) \pi^{+}$has been reconstructed by ATLAS, CMS and LHCb, the former two using $4.9 \mathrm{fb}^{-1}[9,10]$, and the latter $0.37 \mathrm{fb}^{-1}[11]$ of data collected at $\sqrt{s}=7 \mathrm{TeV}$. The mass value measured by $\mathrm{LHCb}$ in this final state is $m\left(B_{c}^{+}\right)=6273.0 \pm 1.3$ (stat) \pm 1.6 (syst) $\mathrm{MeV}$. The decay 
mode $B_{c}^{+} \rightarrow J / \psi\left(\rightarrow \mu^{+} \mu^{-}\right) \pi^{+} \pi^{+} \pi^{-}$has been reconstructed by CMS and LHCb, the former using $4.7 \mathrm{fb}^{-1}$ [10], the latter $0.8 \mathrm{fb}^{-1}$ [12] of data collected at $\sqrt{s}=7 \mathrm{TeV}$. LHCb measured the ratio of the branching ratios $\mathscr{B}\left(B_{c}^{+} \rightarrow J / \psi \pi^{+} \pi^{+} \pi^{-}\right) / \mathscr{B}\left(B_{c}^{+} \rightarrow J / \psi \pi^{+}\right)=2.41 \pm 0.30$ (stat) \pm 0.33 (syst), in agreement with the theoretical predictions. LHCb observed for the first time the decay modes $B_{c}^{+} \rightarrow J / \psi D_{s}^{+}$[13] and $B_{c}^{+} \rightarrow \psi(2 S) \pi^{+}$[14]. The former final state yields the current most precise determination of the $B_{c}^{+}$mass, which is found to be $m\left(B_{c}^{+}\right)=6276.28 \pm 1.44$ (stat) \pm 0.36 (syst) $\mathrm{MeV}$.

\section{Discovery of new states}

The large amount of data collected by the experiments at LHC has led to the observation of predicted resonances and discovery of new states even in the complex environment of hadronic collisions. ATLAS studied the radiative decays of the $\chi_{b}$ to $\Upsilon(1 S)$ and $\Upsilon(2 S)$, and observed for the fist time a new structure centered at a mass of $10.503 \pm 0.005$ (stat) \pm 0.009 (syst) [15], shown in Fig. 1(a), interpreted as the $\chi_{b}(3 P)$ system. The discovery was also confirmed by LHCb, which found a compatible value for the mass [16]. CMS observed a new b baryon [17] via its strong decay into $\Xi_{b}^{-} \pi^{+}$, using $5.3 \mathrm{fb}^{-1}$ of data. The known $\Xi_{b}^{-}$barion was reconstructed via the decay chain $\Xi_{b}^{-} \rightarrow J / \psi\left(\rightarrow \mu^{+} \mu^{-}\right) \Xi^{-}\left(\rightarrow \Lambda^{0} \pi^{-}\right)$, with $\Lambda^{0} \rightarrow p \pi^{-}$, which requires the identification of three displaced decay vertexes. A peak was observed in the distribution of the difference between the mass of the $\Xi_{b}^{-} \pi^{+}$system and the sum of the masses of the $\Xi_{b}^{-}$and $\pi^{+}$, as shown in Fig. 1(b). The fitted value of the difference is $14.84 \pm 0.74$ (stat) \pm 0.28 (syst) $\mathrm{MeV}$, with a significance of the peak exceeding 5 standard deviations. The new state is interpreted as the $J^{P}=3 / 2^{+}$companion of the $\Xi_{b}$. LHCb has been able to observe for the first time the excited states of many hadrons. In particular it observed two $\Lambda_{b}^{0}$ excited states in their decays $\Lambda_{b}^{0} \pi^{+} \pi^{-}$[18], with $\Lambda_{b}^{0} \rightarrow \Lambda_{c}^{+} \pi^{-}, \Lambda_{c}^{+} \rightarrow$ $p K^{-} \pi^{+}$, as shown in Fig. 1(c). The two structures observed are interpreted as the excited states $\Lambda_{b}^{* 0}(5912)$ and $\Lambda_{b}^{* 0}(5920)$, with $m_{\Lambda_{b}^{* 0}(5912)}=5911.97 \pm 0.12 \mathrm{MeV}$ and $m_{\Lambda_{b}^{* 0}(5920)}=5919.77 \pm 0.08$ $\mathrm{MeV}$, and significance $5.2 \sigma$ and $10.2 \sigma$ respectively.

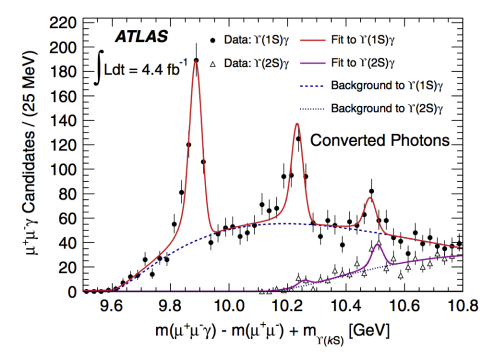

(a)

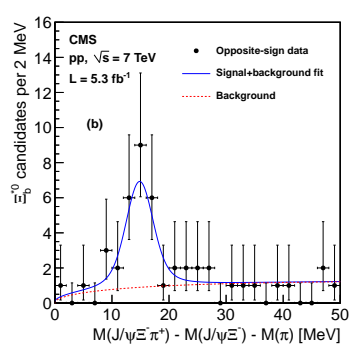

(b)

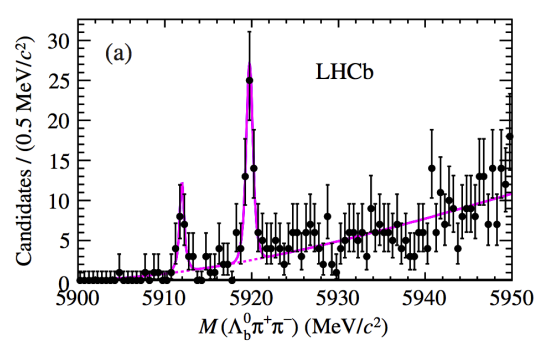

(c)

Figure 1: (a): The mass distributions of $\chi_{b} \rightarrow \Upsilon(k S) \gamma$ candidates reconstructed using photons which have converted in the ATLAS tracker. Solid lines represent the total fit result for each mass window. The dashed lines represent the background components only. (b): Distribution of the difference between the mass of the $\Xi_{b}^{-} \pi^{+}$system and the sum of the masses of the $\Xi_{b}^{-}$and $\pi^{+}$in CMS. In blue a fit to the excess peak. (c): Invariant mass spectrum in LHCb of $\Lambda_{b}^{0} \pi^{+} \pi^{-}$. The points with error bars are the data, the solid line is the fit result, and the dashed line is the background contribution. 


\section{Exotic states}

The surprising discovery of the X(3872) resonance by Belle [19] in 2003 attracted much theoretical interest because it does not match any expected state of quarkonium spectroscopy and has opened the possibilities to more exotic interpretations. The X(3872) has been observed at the LHC both by CMS and LHCb experiments in the decay channel $X(3872) \rightarrow J / \psi \pi^{+} \pi^{-}$. CMS has measured the production cross section and the fraction from b-decays using $4.8 \mathrm{fb}^{-1}$ of 2011 data [20]. The dipion mass spectrum has also been studied by CMS, showing clear evidence of the presence of an intermediate $\rho^{0}$ state. LHCb used $34.7 \mathrm{pb}^{-1}$ of 2010 data to measure the inclusive production cross section and to make the mass measurement $m_{X(3872)}=3871.95 \pm 0.48$ (stat) \pm 0.12 (syst) $\mathrm{MeV}$ [21]. LHCb also determined the quantum numbers to be $J^{P C}=1^{++}$, based on angular correlations in $B^{+} \rightarrow X(3872) K^{+}$decays using $1.0 \mathrm{fb}^{-1}$ of data [22]. The only alternative assignment allowed by previous measurements, $J^{P C}=2^{-+}$, was rejected with a confidence level equivalent to more than eight Gaussian standard deviations. The exotic interpretation is still open, as well as a more conventional assignment to the charmonium level $2^{3} P_{1}$ where the proximity to the $D^{* 0} \overline{D^{0}}$ threshold can have an effect on the observed mass lineshape.

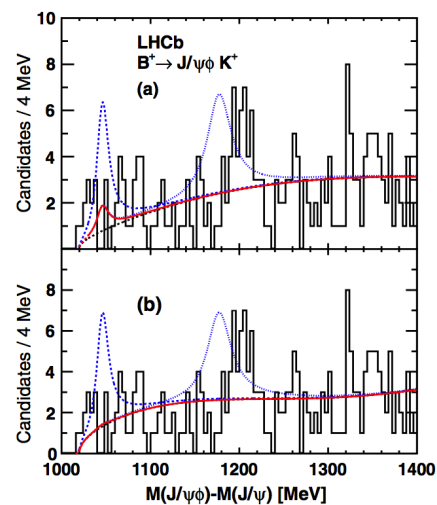

(a)

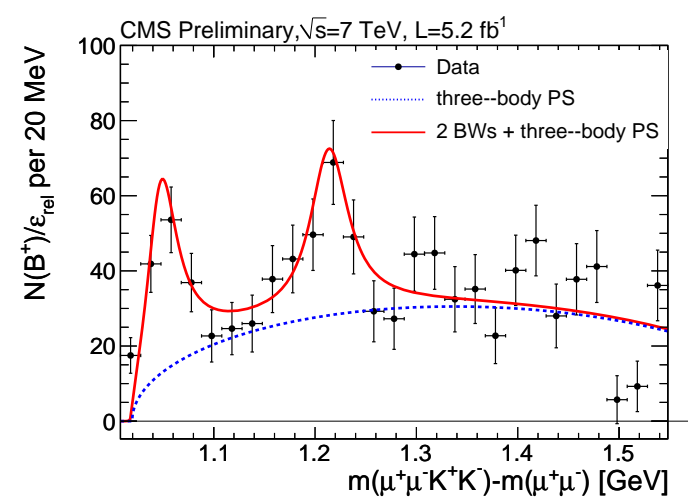

(b)

Figure 2: (a): distribution of the mass difference $M(J / \psi \phi)-M(J / \psi)$ for $B^{+} \rightarrow J / \psi \phi K^{+}$. Fit of $X(4140)$ signal on top of a smooth background is superimposed (solid red line).The dashed blue (dotted blue) line on top illustrates the expected $X(4140)$ signal yield from the CDF measurement. The top and bottom plots differ by the background function. (b): CMS evidence for structures in the $J / \psi \phi$ spectrum in $B^{+} \rightarrow J / \psi \phi K^{+}$ decays.

Other interesting exotic candidates are the two structures decaying to $J / \psi \phi$ observed by CDF in $B^{ \pm} \rightarrow J / \psi \phi K^{ \pm}$decays [23], with one, named X(4140), with a significance above $5 \sigma$. LHCb performed a search in the same decay channel using $0.37 \mathrm{fb}^{-1}$ of data [24]. As shown in Fig. 2(a), no evidence for $X(4140)$ was found, in $2.4 \sigma$ disagreement with the measurement by CDF. CMS produced a preliminary result using $5.2 \mathrm{fb}^{-1}$ of data [25], observing two structures in the mass spectrum, shown in Fig. 2(b). The first one, identified as the X(4140), has a significance above $5 \sigma$ and a fitted mass $m=4148.2 \pm 2.0$ (stat) \pm 4.6 (syst) $\mathrm{MeV}$. Additional studies are ongoing in the collaborations, in particular a complete amplitude analysis, needed to interpret correctly the observed mass spectrum. 


\section{Conclusion}

The experiments at LHC have produced a large amount of results on the spectroscopy of hadrons containing heavy quarks using the data collected in 2010 and 2011. Many studies are ongoing on 2012 data, focusing on the search for and confirmation of exotic or still undiscovered expected states. The trigger strategies are of key importance for the spectroscopy programs in ATLAS and CMS, especially when the operation of LHC will start again at higher values of instantaneous luminosity.

\section{References}

[1] LHCb Collaboration, Precision measurement of D meson mass differences, arXiv:1304.6865.

[2] ATLAS Collaboration, Measurement of the $\Lambda_{b}^{0}$ lifetime and mass in the ATLAS experiment, Phys. Rev. D 87, 032002 (2013).

[3] CMS Collaboration, Measurement of the $\Lambda_{b}^{0}$ lifetime in pp collisions at $\sqrt{s}=7 \mathrm{TeV}$, arXiv:1304.7495.

[4] LHCb Collaboration, Measurement of $\Lambda_{b}^{0}$, $\Xi_{b}^{-}$and $\Omega_{b}^{-}$baryon masses, arXiv:1302.1072.

[5] ATLAS Collaboration, Measurement of the upsilon production in $7 \mathrm{TeV}$ pp collisions at ATLAS, Phys. Rev. D 87, 052004 (2013).

[6] CMS Collaboration, Measurement of the $\Upsilon(1 S), \Upsilon(2 S)$ and $\Upsilon(3 S)$ cross sections in pp collisions at $\sqrt{s}=7 \mathrm{TeV}$, arXiv:1303.5900.

[7] LHCb Collaboration, Measurement of the $\Upsilon$ production in pp collisions at $\sqrt{s}=7 \mathrm{TeV}$, Eur. Phys. J. C (2012) 72:2025.

[8] CMS Collaboration, Measurement of the $\Upsilon(1 S), \Upsilon(2 S)$ and $\Upsilon(3 S)$ cross polarizations in pp collisions at $\sqrt{s}=7 \mathrm{TeV}$, Phys. Rev. Lett. 110, 081802 (2013).

[9] ATLAS Collaboration, Observation of the $B_{c}^{ \pm}$meson in the decay $B_{c}^{ \pm} \rightarrow J / \psi\left(\mu^{+} \mu^{-}\right) \pi^{ \pm}$with the ATLAS detector at the LHC, ATLAS-CONF-2012-028.

[10] CMS Collaboration, Observation of the decay $B_{c}^{+} \rightarrow J / \psi \pi^{+}$and $B_{c}^{+} \rightarrow J / \psi \pi^{+} \pi^{+} \pi^{-}$in pp collisions at $\sqrt{s}=7 \mathrm{TeV}$, CMS PAS BPH-11-003.

[11] LHCb Collaboration, Measurements of $B_{c}^{+}$production and mass with the $B_{c}^{+} \rightarrow J / \psi \pi^{+}$decay, Phys. Rev. Lett. 109, 232001 (2012).

[12] LHCb Collaboration, First observation of the decay $B_{c}^{+} \rightarrow J / \psi \pi^{+} \pi^{+} \pi^{-}$, Phys. Rev. Lett. 108, 251802 (2012).

[13] LHCb Collaboration, Observation of $B_{c}^{+} \rightarrow J / \psi D_{s}^{+}$and $B_{c}^{+} \rightarrow J / \psi D_{s}^{*+}$ decays, arXiv:1304.4530.

[14] LHCb Collaboration, Observation of the decay $B_{c}^{+} \rightarrow \psi(2 S) \pi^{+}$, Phys. Rev. D 87, 071103(R) (2013).

[15] ATLAS Collaboration, Observation of a new $\chi_{b}$ state in radiative transitions to $\Upsilon(1 S)$ and $\Upsilon(2 S)$ at ATLAS, Phys. Rev. Lett. 108, 152001 (2012).

[16] LHCb Collaboration, Observation of the $\chi_{b}$ state at LHCb in pp collisions at $\sqrt{s}=7 \mathrm{TeV}$, LHCb-CONF-2012-020.

[17] CMS Collaboration, Observation of a new $\Xi_{b}$ baryon, Phys. Rev. Lett. 108, 252002 (2012).

[18] LHCb Collaboration, Observation of excited $\Lambda_{b}^{0}$ baryons, Phys. Rev. Lett. 109, 172003 (2012). 
[19] Choi, S. K. and others, Observation of a new narrow charmonium state in exclusive $B^{ \pm} \rightarrow K^{ \pm} \pi^{+} \pi^{-} J / \psi$ decays, Phys. Rev. Lett. 91, 262001 (2003).

[20] CMS Collaboration, Measurement of the X(3872) production cross section via decays to $J / \psi \pi^{+} \pi^{-}$in pp collisions at $\sqrt{s}=7 \mathrm{TeV}$, JHEP 1304 (2013) 154.

[21] LHCb Collaboration, Observation of $X(3872)$ production in pp collisions at $\sqrt{s}=7 \mathrm{TeV}$, Eur. Phys. J. C 72, 1972 (2012).

[22] LHCb Collaboration, Determination of the X(3872) meson quantum numbers, arXiv:1302.6269.

[23] CDF Collaboration, Observation of the $Y(4140)$ structure in the $J / \psi \phi$ mass spectrum in $B^{ \pm} \rightarrow J / \psi \phi K^{ \pm}$decays, arXiv:1101.6058.

[24] LHCb Collaboration, Search for the $X(4140)$ state in $B^{+} \rightarrow J / \psi \phi K^{+}$decays, Phys. Rev. D 85, 091103(R) (2012).

[25] CMS Collaboration, Observation of structures in $J / \psi \phi$ spectrum in exclusive $B^{+} \rightarrow J / \psi \phi K^{+}$decays at $7 \mathrm{TeV}$, CMS-BPH-11-026. 